\section{Combichem leads for asthma}

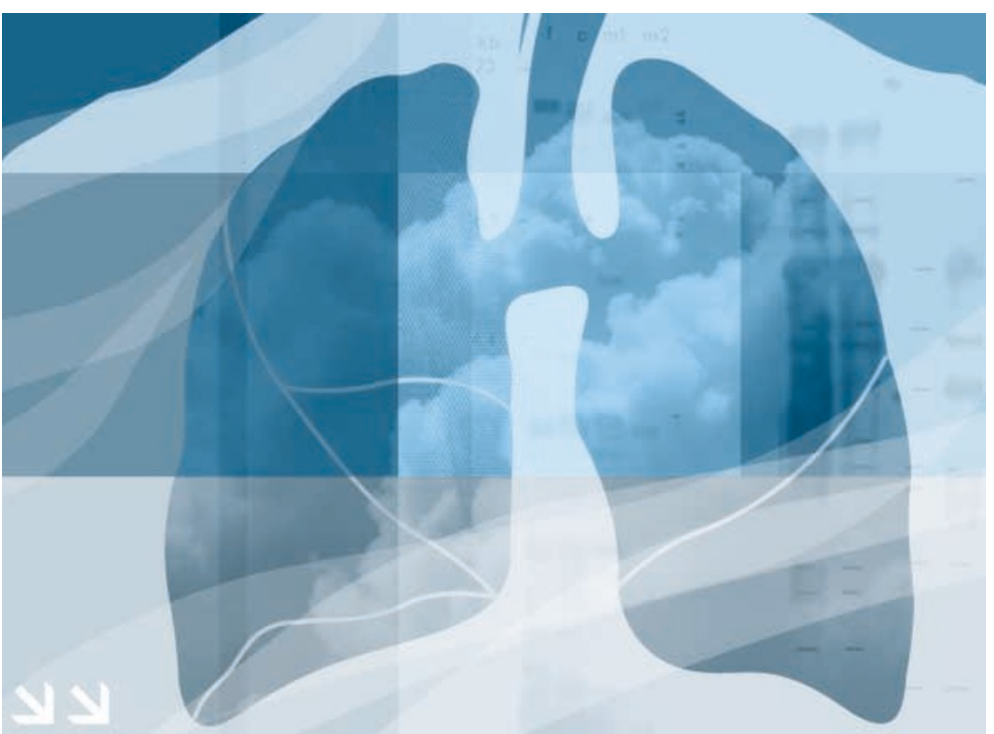

Using chemogenomic techniques, researchers in Seattle describe the design of a novel molecule, PNRI-299, that reduces the inflammation associated with asthma, a disease reaching epidemic proportions worldwide.

Asthmatics experience a complex inflammatory response characterized by airway infiltration of immune cells that undergo respiratory burst activation that releases reactive oxygen species (ROS). Redox-sensitive transcription factors, activator protein (AP)- 1 and nuclear factor- $\kappa \mathrm{B}$, are activated and lead to the expression of certain cytokines, interleukin (IL) 4, IL-5 and IL-13, the molecular hallmarks of asthma. Although the side effects are severe, corticosteroids are able to control the inflammation for many asthmatics. However, a subset of patients respond poorly to these drugs, and their immune cells have increased activity of AP-1.

Michael Kahn and colleagues set out to develop and test a series of designer molecules that might inhibit transcription by redox-sensitive protein AP- 1 and validate it as a therapeutic target for asthma. Taking advantage of the fact that proteinprotein interactions can be mimicked by small molecules bearing similar local structural features, they used a bicyclic template designed to act as a $\beta$-strand, a motif common in redoxsensitive proteins. The template incorporated a functional group, an enedione, which can trap the crucial cysteine residue in the active site of redox proteins. Variation was introduced at two points on the template, by adding different amino acid sidechains, using a highly automated combinatorial approach to generate a library of designer molecules that could be screened for the ability to inhibit AP-1 in human airway epithelial cells.

Once PNRI-299 was identified as an AP-1 inhibitor, the authors tested it in a mouse model of asthma and found that it reduced airway swelling and mucus secretion in the mouse. Furthermore, they discovered that PNRI-299 inhibits the action of an

\section{Polymer-perfect proteins}

Heterogeneity of recombinant protein drugs - for example, owing to variations in glycosylation pattern - results in undesirable variability of potency and pharmacokinetic properties, and has been associated with serious immune reactions. Modifying recombinant proteins with conventional polymers, such as polyethylene glycol, can address some of these shortcomings, but there is still some heterogeneity both in the polymers attached and the site of attachment, leading to variability in biological properties. Now, as described in Science, Kochendoerfer and colleagues have addressed these problems by using total chemical synthesis to produce a homogeneous population of a monodisperse polymer-modified synthetic erythropoiesis protein (SEP), a version of the highly successful protein drug human erythropoetin (Epo).

Epo is a glycoprotein hormone that regulates the proliferation and development of red blood cells through the Epo receptor, and recombinant Epo is widely used in the treatment of anaemia. The authors designed SEP - which consists of a 166-amino-acid chain similar to the sequence of Epo, with two precisely designed polymers attached specifically to two of the glycosylation sites of Epoto be a potent activator of the Epo receptor. Chemical synthesis of SEP begins with the generation of four separate peptide fragments by standard solid-phase peptide synthesis. Using previously established ligation techniques, the branched polymer moiety is then attached to a specific lysine residue on two of the four fragments, before all of the fragments are assembled into the complete, precisely defined protein-polymer construct.

So, do the unique properties of SEP confer an advantage over proteins that are modified with conventional polymers? Following folding and purification of the protein-polymer construct, more than 100 $\mathrm{mg}$ of SEP - representing yields for each step of the synthesis pathway of between 15 and $50 \%$ - was available for testing of physicochemical and biological properties. The biological activity of SEP on the Epo receptor was confirmed in cell-proliferation assays, and the in vitro haematopoietic activity of SEP was shown to be similar to that of Epo. This contrasts markedly with recombinant growth factors that are modified with conventional polymers, whose in vitro activity can be drastically reduced relative to their unmodified counterparts.
But the most exciting demonstration of SEP's superior clinical potential came from in vivo tests in several animal models - under two different dosing regimens, SEP consistently outperformed Epo in terms of haematopoietic activity. Pharmacokinetic data showed that this increased in vivo potency is due to SEP's longevity in the circulation, which exceeds that of Epo by 2-3 times. The authors suggest that the presence of the precision polymer prolongs the lifetime of SEP in the circulation. This prolonged efficacy is a very favourable characteristic for intravenously administered pharmaceuticals to have - the longer a drug persists in a patient's system, the fewer injections are required, and the more widely used a drug is likely to be. So, not only is the development of SEP a potentially promising advance over Epo in the treatment of anaemia, but more generally, the approach used could be a valuable tool for the development of new protein therapeutics with improved biological properties.

Suzanne Farley

(2) References and links ORIGINAL RESEARCH PAPER Kochendoerfer, G. G. et al. Design and chemical synthesis of a homogenous polymermodified erythropoiesis protein. Science 229, 884-887 (2003) FURTHER READING Kochendoerfer, G. G. Chemical protein synthesis methods in drug discovery. Curr. Opin. Drug Discov. Devel. 4, 205-214 (2001) WEB SITE

Encyclopedia of Life Sciences: http://www.els.net/ Anaemia | haematopoiesis | haematopoietic growth factors 
AP-1 activator, Ref-1. They predicted that because regulation of IL- 5 and IL-13 is independent of AP- 1 activation, the expression of these molecules would be unchanged in the presence of PNRI-299. As expected, IL-4 expression, but not that of IL-5 and IL-13, was decreased.

Chemogenomics is a powerful approach for the generation of drug leads. In the case of inflammatory disorders, it can be used to validate a range of redox superfamily members as important targets for drug design.

Melanie Brazil

(2) References and links ORIGINAL RESEARCH PAPER Nguyen, C. et al. Chemogenomic identification of Ref-1/AP-1 as a therapeutic target for asthma. Proc. Natl Acad. Sci. USA 100, 1169-1173 (2003)

FURTHER READING Ramstrom, O. \& Lehn, J-M. Drug discovery by dynamic combinatorial libraries. Nature Rev. Drug Discov. 1, 26-36 (2002) | Barnes, P. J. Transcription factors and asthma. Eur. Respir. J. 12, 221-234 (1998) | Corry, D. B. Emerging immune targets for the treatment of allergic asthma. Nature Rev. Drug Discov. 1, 55-64 (2002) | Holgate, S. T. The epidemic of allergy and asthma. Nature 402, (Suppl. 6760), B2-B4 (1999) WEB SITE Encyclopedia of Life Sciences: http://www.els.net Asthma

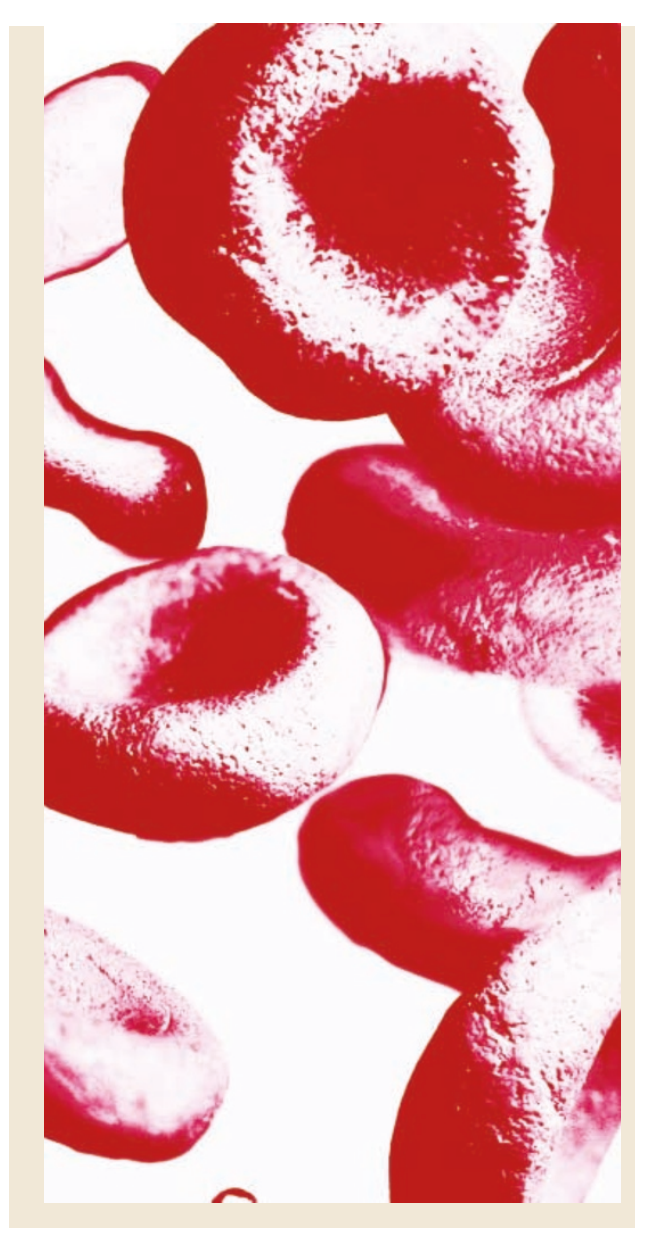

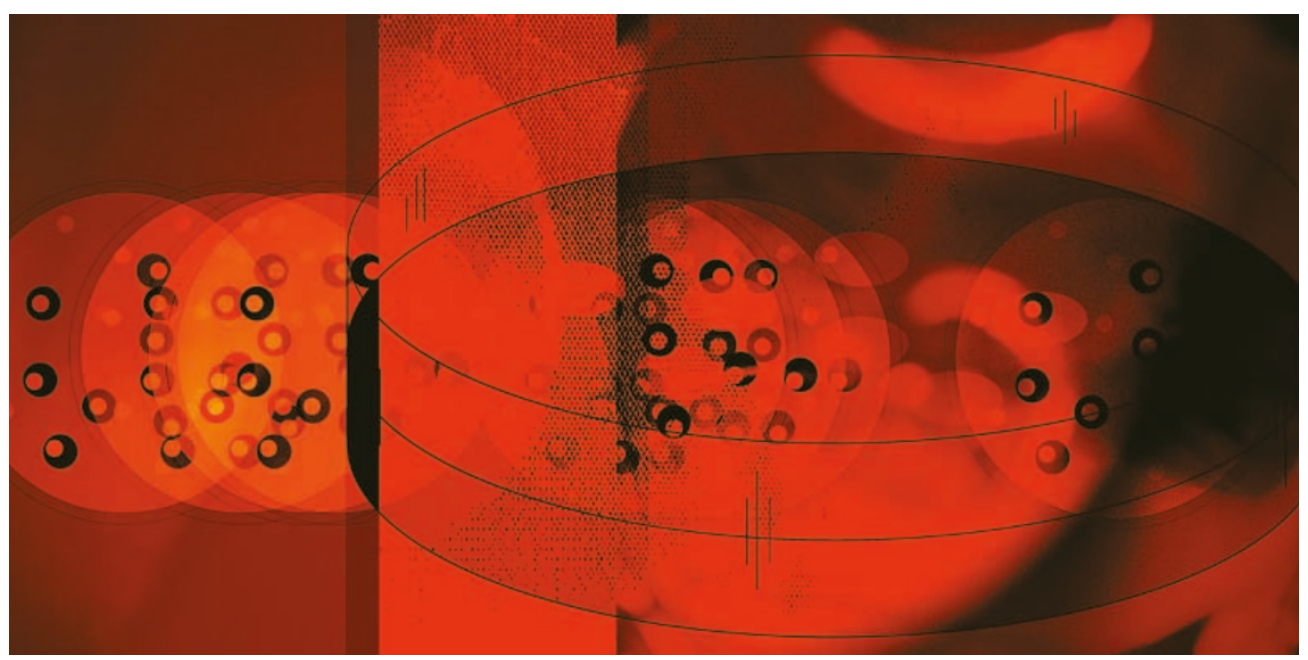

\section{ANTICOAGULANTS}

\section{The clot thickens}

The occlusion of blood vessels by abnormal blood clots continues to be the biggest cause of disability and death in the Western world. So it is no surprise that much effort has been directed towards finding modulators of proteins in the blood-clotting cascade, particularly the protease thrombin. Now, writing in Chemistry \& Biology, Famulok and colleagues report using messenger RNA (mRNA)-display - an in vitro display technique that, like phage and ribosome display, is used to identify peptides with specific desired features - to isolate novel peptides that bind human $\alpha$-thrombin.

Thrombin, which is the main effector protease in the coagulation cascade, interacts with many other proteins, and is recognized by a wide range of natural macromolecules at a variety of epitopes, for example, by heparin, which is a widely used anticoagulant. Famulok and colleagues wanted to find novel peptides that bound $\alpha$-thrombin, and set about this by generating a DNA library encoding a 35-aminoacid peptide containing a randomized 15 -amino acid sequence within it ( 15 amino acids is roughly the length of natural thrombin-binding motifs). To produce the mRNA-display library, the DNA sequences were translated in an in vitro system so that the translated protein remained covalently attached to its mRNA, which aids the recovery of mRNA sequences that code for proteins of interest. Although a randomized region of 15 amino acids can theoretically produce $3 \times 10^{19}$ different peptides, only $1.2 \times$ $10^{11}$ different peptides were obtained for the study, a number comparable to the total repertoire of human antibodies.
The randomly generated peptides were then enriched for peptides that bind to $\alpha$-thrombin through rounds of selection and amplification, in which a thrombin-derivatized matrix was used to bind peptides, so that non-binding peptides could be eluted off. Those peptides that bound were then recovered from the matrix and then subjected to another round of binding in more stringent conditions. From 72 clones randomly selected for analysis, 45 different sequences were obtained, of which more than $60 \%$ contained the four-aminoacid sequence motif DPGR. It is notable that none of the sequences shared any sequence similarity with natural thrombin-binding proteins. Two clones were chosen for binding studies from which free peptides were derived, and both were able to bind to a thrombin-derivatized matrix, whereas control peptides did not. By comparing the competitive binding of the two peptides with molecules that are known to bind to two epitopes on $\alpha$-thrombin, it was determined that the most likely binding site of the novel peptides was near or at exosite-1 (the fibrinogen-recognition exosite).

Functional studies of both peptides revealed that they were able to prolong blood-clotting in vitro, although less well than hirudin, an anticoagulant peptide derived from leeches. These findings reiterate the general utility of mRNA display approaches in elucidating protein-protein interactions, and specifically indicate that the technology could be useful in identifying novel, non-natural peptides that modulate thrombin or other of the myriad proteins in the blood-clotting cascade, which might then serve as a basis for developing therapeutic molecules that target this important group of proteins.

Daniel Jones

(0) References and links

ORIGINAL RESEARCH PAPER Raffler, N. A., Schneider-Mergener, J. \& Famulok, M. A novel class of small functional peptides that bind and inhibit human $\alpha$-thrombin isolated by mRNA display. Chem. Biol. 10 69-79 (2003)

FURTHER READING Wilson, D. S., Anthony, D. K. \& Szostak, J. W. The use of mRNA display to select high-affinity protein-binding peptides. Proc. Natl Acad. Sci. USA 98, 3750-3755 (2001) 